\title{
Shunt placement after cyst fenestration for middle cranial fossa arachnoid cysts in children
}

\author{
Tomomi Kimiwada, MD, PhD, ${ }^{1}$ Toshiaki Hayashi, MD, PhD, ${ }^{2}$ Ayumi Narisawa, MD, PhD, ${ }^{2}$ \\ Reizo Shirane, MD, PhD, ${ }^{1}$ and Teiji Tominaga, MD, PhD ${ }^{3}$
}

'Department of Neurosurgery, Miyagi Children's Hospital; ${ }^{2}$ Department of Neurosurgery, Sendai City Hospital; and ${ }^{3}$ Department of Neurosurgery, Tohoku University Graduate School of Medicine, Sendai, Japan

\begin{abstract}
OBJECT Some pediatric patients with middle cranial fossa arachnoid cysts present with symptoms of increased intracranial pressure (ICP) and require shunt placement after a cyst fenestration. However, factors concerning increased ICP after fenestration followed by shunt placement have not been elucidated. This study evaluated factors that are associated with shunt placement following cyst fenestration in pediatric patients with middle cranial fossa arachnoid cysts.

METHODS Twenty-six pediatric patients with middle cranial fossa arachnoid cysts who were surgically treated at a single institution between 2004 and 2013 were retrospectively identified. The surgical indications for middle cranial fossa arachnoid cysts were as follows: 1) arachnoid cysts associated with symptoms such as headache and abnormally enlarging head circumference; 2) progressively expanding arachnoid cysts; and 3) large arachnoid cysts such as Galassi Type III. A cyst fenestration was performed as a first-line treatment, and shunt placement was required if symptoms associated with increased ICP were found following fenestration. The risk factors evaluated included age, sex, presenting symptoms, the presence of head enlargement, progressive cyst expansion, and subdural hematoma/hygroma.
\end{abstract}

RESULTS Four patients (15.4\%) required shunt placement after cyst fenestration. Younger age, abnormal head enlargement, and progressive cyst expansion before fenestration were significantly associated with the need for shunt placement following fenestration. Arachnoid cysts decreased in size in 22 patients (84.6\%) after fenestration and/or shunt placement. The presence of symptoms was not associated with postoperative cyst size in this study.

CONCLUSIONS In this study, younger age, abnormal head enlargement, and progressive cyst expansion were risk factors for shunt placement after cyst fenestration in pediatric patients with middle cranial fossa arachnoid cysts. It is important to consider that cyst fenestration may not be effective because of a latent derangement of CSF circulation in patients with these risk factors.

http://thejns.org/doi/abs/10.3171/2015.3.PEDS14573

KEY WORDS middle fossa arachnoid cyst; shunt placement; fenestration; macrocephaly; congenital

$\mathrm{T}$ HE natural history of middle cranial fossa arachnoid cysts in children is not well defined, and the optimal treatment remains controversial. However, there appears to be some consensus that asymptomatic arachnoid cysts should not be treated. Recently, there has been an increase in the number of incidentally diagnosed arachnoid cysts with the increased use of MRI and CT. Therefore, clinicians face difficulties in selecting treatment strategies because the symptoms of arachnoid cysts are vague in many clinical situations and the risks of surgery are not negligible. Most arachnoid cysts remain static and clinically silent, whereas others may spontaneously disappear, expand, rupture, exert mass effects, or promote hydrocephalus. ${ }^{2,4,10,11}$ For symptomatic cases, many different surgical procedures have been proposed, including cyst fenestration using microscopy or endoscopy and/or shunt placement. ${ }^{6,7,16,18}$

It is known that some patients with middle cranial fossa arachnoid cysts present with increased intracranial pressure (ICP) and hydrocephalus and require shunt placement

ABBREVIATION ICP = intracranial pressure.

SUBMITTED October 17, 2014. ACCEPTED March 24, 2015.

INCLUDE WHEN CITING Published online July 31, 2015; DOI: 10.3171/2015.3.PEDS14573.

DISCLOSURE The authors report no conflict of interest concerning the materials or methods used in this study or the findings specified in this paper. 
after a successful cyst fenestration; ${ }^{9,11,12,15}$ however, there is no clear explanation for the pathogenesis of subsequent intracranial hypertension and the risk factors for shunt placement after fenestration. The middle cranial fossa is the most common location for intracranial arachnoid cysts, and obstructive hydrocephalus is uncommon. Thus, it is speculated that a latent derangement of CSF circulation becomes clinically evident following cyst fenestration.

In our study, we retrospectively reviewed a single-institution experience with 26 pediatric patients (21 symptomatic, 5 asymptomatic with large cysts) who underwent surgical treatment of middle cranial fossa arachnoid cysts during a 9-year period and evaluated the risk factors for shunt placement following a successful cyst fenestration. In addition, we discussed the optimal surgical indications for the treatment of middle cranial fossa arachnoid cysts in children.

\section{Methods \\ Patient Population}

From the retrospective analysis of 35 endoscopic or microsurgical cyst fenestrations performed for various intracranial arachnoid cysts between April 2004 and March 2013 in the Department of Neurosurgery, Miyagi Children's Hospital, 26 pediatric patients (defined as age $<18$ years) with middle cranial fossa arachnoid cysts were studied in this report. Patients with a follow-up period of less than 1 year were excluded. Our surgical indications for middle cranial fossa arachnoid cysts were as follows: 1) arachnoid cysts associated with symptoms such as headache and abnormally enlarging head circumference; 2) progressively expanding arachnoid cysts; and 3) large arachnoid cysts such as Galassi Type III.

\section{Data Collection}

Preoperatively, we recorded age, sex, presenting symptoms, and the presence of increased head circumference, progressive cyst expansion, and subdural hematoma/hygroma for each patient. Abnormal head enlargement was defined as a head circumference greater than 2 standard deviations above the mean or greater than the 98th percentile. The radiological studies, including CT and MRI, were evaluated for any changes in cyst size, usually in the axial plane; cyst enlargement was determined by a consistent numerical increase in size in at least 2 consecutive measurements. Statistical analysis was performed using JMP software (version 11, SAS Institute Inc.).

\section{Surgical Treatment}

All patients were operated on as a primary surgery without any history of microsurgical and/or endoscopic treatment, or any shunt placement. First, we performed cyst fenestration to avoid shunt-related complications such as shunt malfunction, infection, over-shunting, and shunt dependency. ${ }^{13} \mathrm{~A}$ drain catheter was inserted in only 1 patient (Case 25). Medical treatments including steroid and/ or glycerol administration were not performed preoperatively in all of the patients. The patients were clinically assessed postoperatively regarding any improvement or worsening of preoperative symptoms, the occurrence of intracranial hypertension requiring subsequent shunt placement, and changes in cyst volume. All patients were evaluated via CT and/or MRI studies pre- and postoperatively.

\section{Results}

\section{Patient Characteristics}

There were 26 patients with middle cranial fossa arachnoid cysts at the time of fenestration (Table 1). This patient population included 17 males and 9 females, with an age range from 9 months to 15 years (median 7.8 years). Clinical manifestations on diagnosis included intermittent vomiting and/or headache in 15 patients, large asymptomatic arachnoid cysts in 7 patients, head enlargement in 2 patients, and developmental delays in 2 patients. In a detailed analysis of head circumference growth curves, 8 patients (including 2 asymptomatic patients [Cases 15 and 24]) displayed abnormally enlarging head circumference greater than 2 standard deviations above the mean or greater than the 98th percentile, meaning that this study actually included 5 asymptomatic patients. Preoperative cyst expansion was found in 4 patients (age range 11 months to 2 years). Younger age was significantly correlated with progressive cyst expansion in patients with untreated middle cranial fossa arachnoid cysts (mean age 1.6 vs 9.0 years, respectively; $p<0.01$, Wilcoxon signedrank test), whereas no patients older than 3 years exhibited cyst expansion during follow-up. Subdural hematoma/hygroma was recognized in 4 patients at the time of initial diagnosis (age range 3-5 years). These clinical characteristics are shown in Table 1.

\section{Surgical Treatment}

Microsurgical cyst fenestration was performed in 25 patients with middle cranial fossa arachnoid cysts. An external subdural drain catheter was inserted in 1 patient (Case 25) simultaneously with fenestration because this patient exhibited subdural hygroma and severe papilledema before fenestration. In brief, under general anesthesia, a 5-cm-long linear skin incision was made behind the hairline, and a $3-\mathrm{cm}$ cranial flap was removed. At this stage, the microscope was brought into the surgical field, and the dura mater was opened and sutured with the outer arachnoid membranes to prevent postoperative hygroma. Sharp dissection of the deep inner arachnoid membranes and the membrane of Liliequist was performed using microscissors to communicate with the deep basal cisterns. Endoscopic fenestration was performed in 1 patient (Case 22). Four patients (Cases 5, 7, 13, and 25) continued to exhibit and/or developed signs of raised ICP. Three patients (Cases 5, 7, and 13) exhibited markedly increased subcutaneous CSF effusion, and the other patient (Case 25) displayed CSF drainage exceeding $300 \mathrm{ml} /$ day after microsurgical fenestration. These 4 patients required subsequent cystoperitoneal shunting (age range 11 months to 4 years) irrespective of fenestration. Shunt placement was performed within 1 month after fenestration (range 6 days to 1 month) with Medtronic Strata programmable valves for 3 patients (Cases 5, 7, and 25) and a Medtronic medi- 
TABLE 1. Summary of clinical presentation in 26 pediatric patients with middle cranial fossa arachnoid cysts

\begin{tabular}{|c|c|c|c|c|c|c|c|}
\hline $\begin{array}{l}\text { Case } \\
\text { No. }\end{array}$ & $\begin{array}{l}\text { Age (yrs), } \\
\text { Sex }\end{array}$ & Preop Symptoms & $\begin{array}{c}\text { Head } \\
\text { Enlargement }\end{array}$ & $\begin{array}{c}\text { Cyst } \\
\text { Expansion }\end{array}$ & Shunt & $\begin{array}{c}\text { Postop } \\
\text { Symptoms }\end{array}$ & Postop Cyst Size \\
\hline 1 & 4.34, M & $\mathrm{HA}, \mathrm{CSH}$ & Yes & No & No & Disappeared & Reduced \\
\hline 2 & $5.38, \mathrm{~F}$ & $\mathrm{HA}, \mathrm{CSH}$ & No & No & No & Disappeared & Unchanged \\
\hline 3 & $9.02, \mathrm{M}$ & None & No & No & No & None & Almost disappeared \\
\hline 4 & 14.36, M & $\mathrm{HA}$ & Yes & No & No & Disappeared & Reduced \\
\hline $5^{*}$ & $1.68, \mathrm{M}$ & Macrocephaly & Yes & Yes & Yes & Disappeared & Hygroma \\
\hline 6 & $5.95, \mathrm{M}$ & None & No & No & No & None & Reduced \\
\hline $7^{*}$ & $1.12, \mathrm{M}$ & Macrocephaly & Yes & Yes & Yes & Improved & Reduced \\
\hline 8 & $9.18, M$ & $\mathrm{HA}$, nausea & No & No & No & Disappeared & Reduced \\
\hline 9 & $15.5, \mathrm{~F}$ & HA, vomiting & No & No & No & Disappeared & Reduced \\
\hline 10 & $2.53, \mathrm{~F}$ & $\mathrm{DD}$ & No & Yes & No & Not evaluable & Almost disappeared \\
\hline 11 & $14.71, \mathrm{~F}$ & $\mathrm{HA}$ & No & No & No & Disappeared & Almost disappeared \\
\hline 12 & 7.18, M & None & No & No & No & None & Almost disappeared \\
\hline $13^{*}$ & 0.91, M & $\mathrm{DD}$ & No & Yes & Yes & Not evaluable & Reduced \\
\hline 14 & $14.27, \mathrm{~F}$ & HA, papilledema & No & No & No & Improved & Almost disappeared \\
\hline 15 & $5.51, \mathrm{M}$ & None (macrocephaly) & Yes & No & No & None & Reduced \\
\hline 16 & 8.63, M & $\mathrm{HA}$ & No & No & No & Disappeared & Almost disappeared \\
\hline 17 & $15.92, \mathrm{~F}$ & $\mathrm{HA}$ & No & No & No & Disappeared & Reduced \\
\hline 18 & $8.50, \mathrm{M}$ & None & No & No & No & None & Reduced \\
\hline 19 & $6.22, \mathrm{M}$ & Vomiting & No & No & No & Disappeared & Hygroma \\
\hline 20 & $11.06, \mathrm{~F}$ & $\mathrm{HA}$ & No & No & No & Disappeared & Reduced \\
\hline 21 & $3.30, \mathrm{~F}$ & $\mathrm{HA}$, vomiting, $\mathrm{CSH}$ & Yes & No & No & Disappeared & Reduced \\
\hline 22 & $14.55, \mathrm{M}$ & $\mathrm{HA}$ & No & No & No & Disappeared & Unchanged \\
\hline 23 & $0.78, \mathrm{M}$ & Vomiting & No & No & No & Disappeared & Reduced \\
\hline 24 & $9.46, \mathrm{M}$ & None (macrocephaly) & Yes & No & No & None & Reduced \\
\hline $25^{*}$ & $4.61, \mathrm{~F}$ & $\mathrm{HA}$, vomiting, papilledema, CSH & Yes & No & Yes & Disappeared & Reduced \\
\hline 26 & $9.84, \mathrm{M}$ & None & No & No & No & None & Almost disappeared \\
\hline
\end{tabular}

$\mathrm{CSH}=$ chronic subdural hematoma/hygroma; $\mathrm{DD}=$ developmental delay; $\mathrm{HA}=$ headache.

* Cases 5, 7, 13, and 25 required shunt placement after cyst fenestration.

um-pressure nonprogrammable valve for 1 patient (Case 13). No asymptomatic patients underwent shunt placement. Statistical analysis identified younger age (mean 2.1 vs 8.9 years old, respectively; $\mathrm{p}<0.01$, Wilcoxon signedrank test; Fig. 1), abnormal head enlargement (37.5\% vs $5.6 \%$, respectively; $\mathrm{p}<0.05$, chi-square test; Fig. 2), and progressive cyst expansion (75.0\% vs $4.6 \%$, respectively; p $<0.001$, chi-square test; Fig. 3) before fenestration as significantly correlated with the need for shunt placement in pediatric patients with middle cranial fossa arachnoid cysts.

\section{Postoperative Complications and Outcome}

There were no perioperative deaths or permanent neurological defects. Two patients (Cases 5 and 19) developed ipsilateral subdural hygroma after microsurgical fenestration, which gradually diminished. There was 1 case of shunt infection during the follow-up period.

The mean follow-up period was 37.3 months. There were no excluded patients for the assessment of the shunt placement. Intermittent vomiting, headache, and head enlargement because of increased ICP were resolved or improved in all patients. Two patients who had develop- mental delays did not display any improvement (Cases 10 and 13).

Radiologically, arachnoid cysts were reduced in size $(<$ $50 \%)$ or nearly resolved $(<90 \%)$ in 22 patients $(84.6 \%)$ after fenestration and/or shunt placement, whereas in the remaining 2 patients (Cases 2 and 22, 7.7\%), there was no postoperative change in cyst size. Because of the subdural hygroma, radiological evaluation could not be performed in 2 patients (Cases 5 and 19). Regarding the 5 asymptomatic patients (Cases 3, 6, 12, 18, and 26), the arachnoid cysts had almost disappeared in 3 patients and had decreased in size in 2 patients. The presence of symptoms was not statistically associated with cyst size after fenestration in pediatric patients with middle cranial fossa arachnoid cysts. In addition, of the 4 patients who required shunt placement, 2 (Cases 7 and 25) showed enlargement of the ventricle size after cyst fenestration because mass effects on adjacent brain parenchyma by arachnoid cysts were reduced, but their enlarged ventricles did not decrease in size after shunt placement (Fig. 4). The other 2 patients (Cases 5 and 13) did not show any changes in ventricle size after fenestration and shunting. Only 1 patient (Case 25) suffered from vomiting 1 year after shunt 


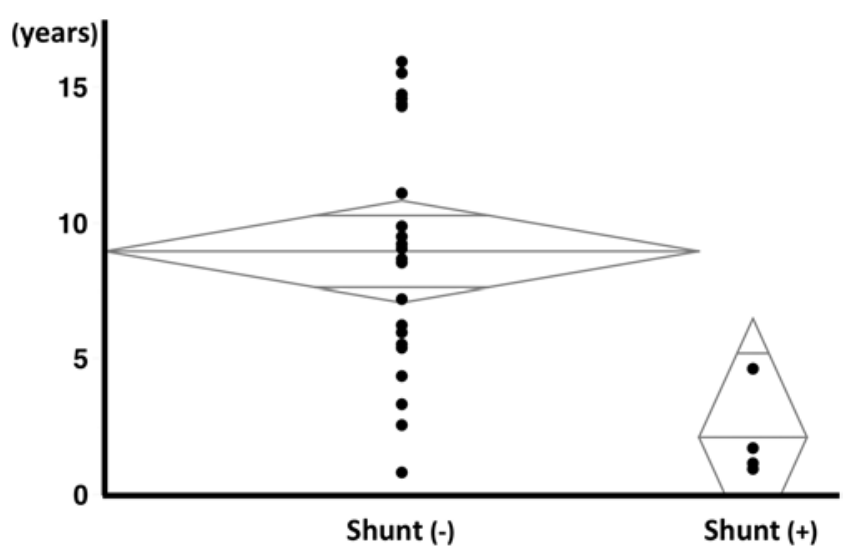

FIG. 1. Scatterplot illustrating the prevalence of pediatric patients with middle cranial fossa arachnoid cysts (by age in years) who did or did not require shunt placement after cyst fenestration. Younger age was a statistically significant risk factor for shunt placement after fenestration (mean age 2.1 vs 8.9 years, respectively; $p<0.01$, Wilcoxon signed-rank test). Mean represented by the middle horizontal line in each category.

placement, and we assessed her shunt patency with a shunt contrast study. Her shunt catheter was patent over the entire length of the shunt.

\section{Illustrative Cases}

\section{Case 7}

A 1-year-old boy was presented to our clinic with a history of progressive head enlargement (from $45.4 \mathrm{~cm}$ at 4 months to $51.2 \mathrm{~cm}$ at 9 months; Fig. 4A). A head CT scan uncovered a large left middle cranial fossa arachnoid cyst (Fig. 4B). He underwent microsurgical cyst fenestration because he was symptomatic. After surgery, he was fretful and irritable, and a markedly increasing subcutaneous CSF effusion at the incision site developed in a pseudomeningocele-like manner. Because his clinical characteristics were consistent with elevated ICP, cystoperitoneal shunt placement was performed 1 week after fenestration. The symptoms associated with increased ICP and the

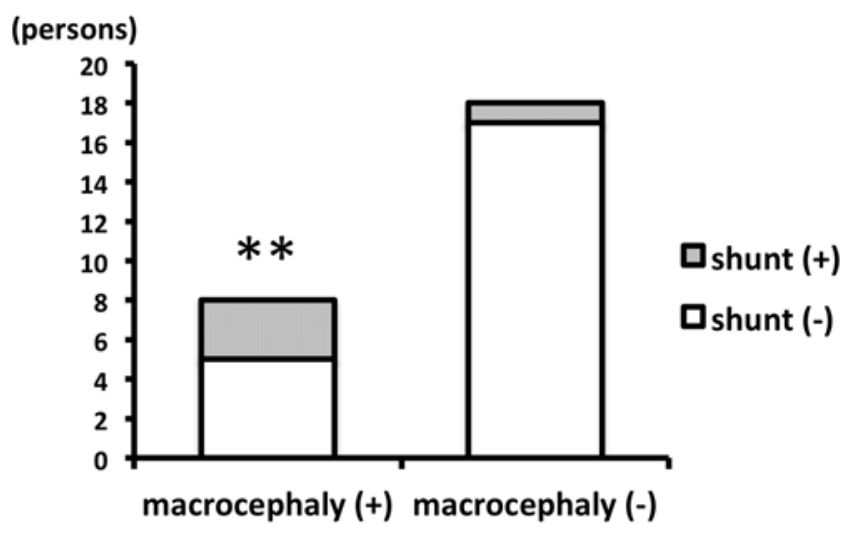

FIG. 2. Bar graph illustrating the number of pediatric patients with middle cranial fossa arachnoid cysts with (+) or without (-) macrocephaly, and with (+) or without (-) shunt placement. Macrocephaly was a significant risk factor for shunt placement after cyst fenestration (37.5\% vs $5.6 \%$, respectively). ${ }^{* *} p<0.05$, chi-square test.

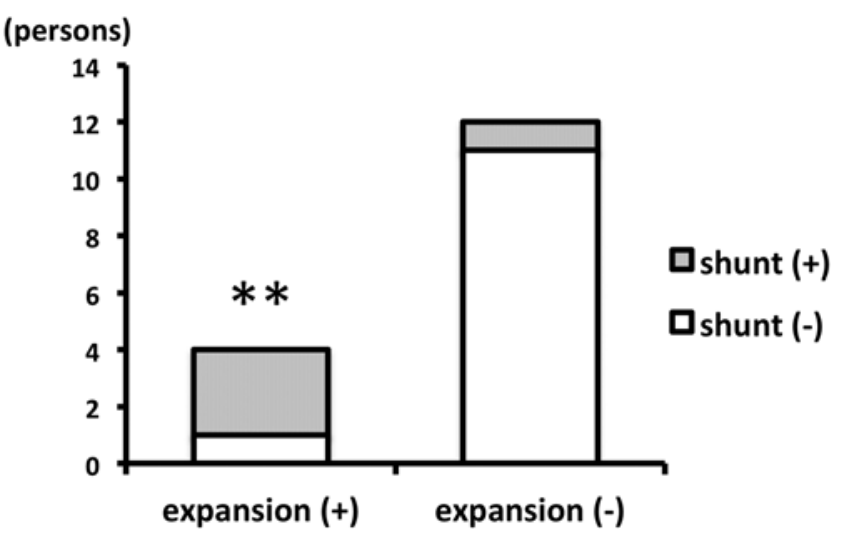

FIG. 3. Bar graph illustrating the number of pediatric patients with middle cranial fossa arachnoid cysts with (+) or without (-) progressive cyst expansion and with (+) or without (-) shunt placement. Progressive cyst expansion was a significant risk factor for shunt placement after cyst fenestration $\left(75.0 \%\right.$ vs $4.6 \%$, respectively). ${ }^{* *} p<0.001$, chi-square test.

preexisting arachnoid cyst resolved after shunt placement (Fig. 4C).

\section{Case 18}

An 8-year-old boy was presented to our clinic after hitting his head in a car accident. Clinically, he was normal and underwent a CT examination, which disclosed a huge left middle cranial fossa arachnoid cyst classified as Galassi Type III (Fig. 5B). Because of the large cyst size, microsurgical fenestration was performed without complications (Fig. 5A). The arachnoid cyst decreased in size dramatically after the fenestration (Fig. 5C).

\section{Discussion}

\section{Shunt Placement After Cyst Fenestration}

In this study we found that younger age, head enlargement, and cyst expansion were risk factors for shunt placement following cyst fenestration in patients with middle cranial fossa arachnoid cysts. The pathogenesis explaining why a latent derangement of CSF circulation becomes clinically evident after fenestration has not been clearly elucidated. However, some patients with middle cranial fossa arachnoid cysts experience an accumulation of CSF and increased ICP after cyst fenestration. It has been reported that the subsequent shunt placements after fenestration were required in $4.0 \%-24.1 \%$ of the patients with middle cranial fossa arachnoid cysts. ${ }^{12,15}$ In our study, 4 pediatric patients $(15.4 \%)$ required CSF conversion with shunt placement after cyst fenestration. Among these patients, 3 displayed increasing subcutaneous CSF effusion in a pseudomeningocele-like manner at the incision site, suggesting elevated ICP. For the remaining patient, a CSF drain catheter was placed at the same time as fenestration because of severe preoperative papilledema. Because more than $300 \mathrm{ml}$ of CSF was drained from the drain catheter each day, cystoperitoneal shunting was performed following fenestration in this patient. The indication of the subsequent shunt placement after fenestration is important but still controversial. We should always consider that it might be a transient overproduction of the CSF due 

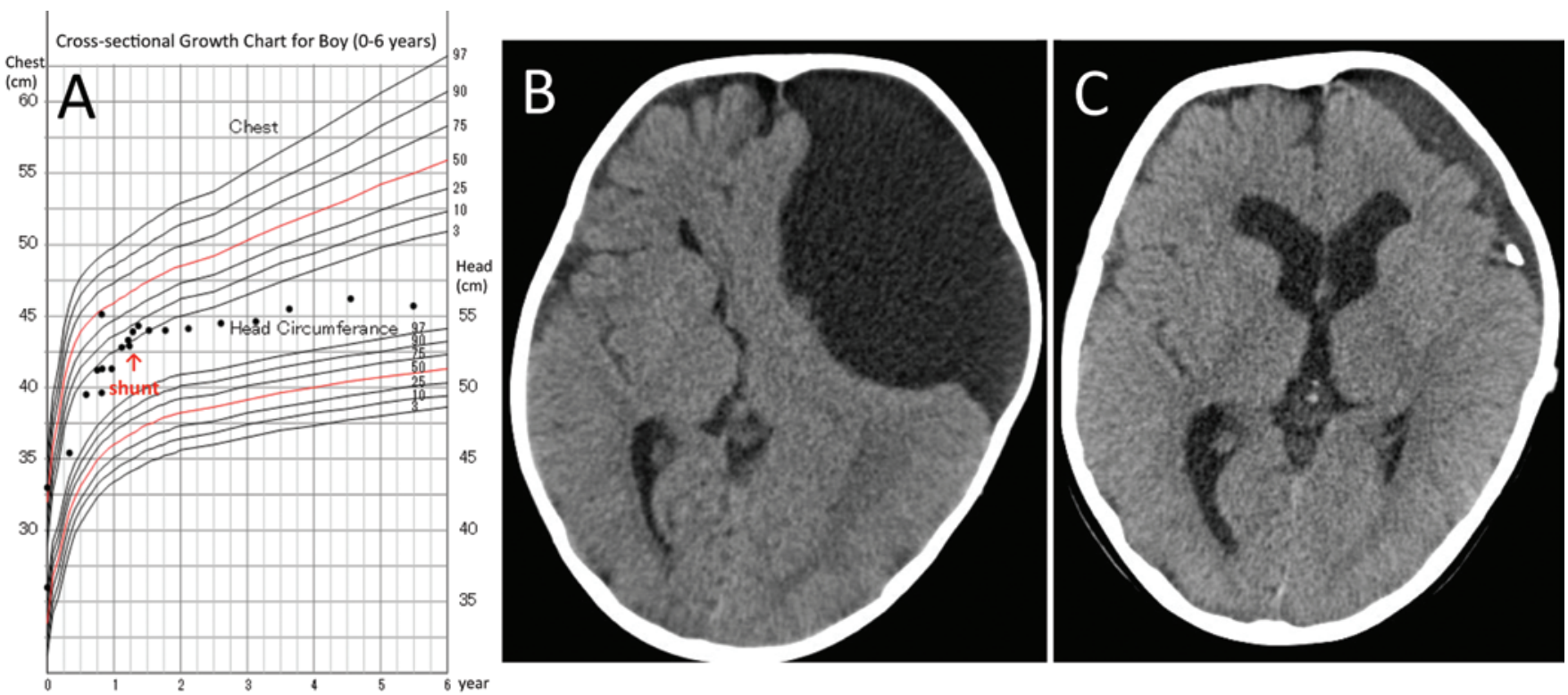

FIG. 4. Case 7. A: Plots of serial head circumference for a 1-year-old boy, who presented with an abnormally enlarging head. The enlargement of his head circumference stopped and plateaued after shunt insertion. B: Preoperative axial CT image illustrating a large left middle cranial fossa arachnoid cyst (Galassi Type III). C: Axial CT image after cyst fenestration followed by shunt placement. Note that the slight ventriculomegaly confirmed the successful cyst fenestration. Figure is available in color online only.

to some factors such as operative stress or aseptic and/or chemical meningitis.

There are few reports regarding risk factors for shunt placement following cyst fenestration in pediatric patients with middle cranial fossa arachnoid cysts. Levy et al. reported 40 children with middle cranial fossa arachnoid cysts treated in their hospital, including 6 patients with ventriculomegaly, 9 patients with macrocephaly but without ventriculomegaly, and 25 patients with normocephaly. All 6 patients with ventriculomegaly required both fenestration and shunt placement. In total, 5 of 9 patients with macrocephaly but without ventriculomegaly, and only 1 patient with normocephaly, underwent initial cyst fenestration and subsequent shunt placement. ${ }^{11}$ Our findings concur with theirs, and these results suggest that patients with middle cranial fossa arachnoid cysts with abnormally enlarging head circumference harbor a latent aberration of CSF circulation and require shunt placement.

Younger age and cyst enlargement before fenestration were also risk factors for shunt placement after fenestration in pediatric patients with middle cranial fossa arachnoid cysts. In our study, all patients with cyst expansion before surgery were less than 2 years old, and a younger age itself was significantly correlated with cyst expansion. Al-Holou et al. performed a survey of the natural history of 111 arachnoid cysts in pediatric patients, of which 11 increased in size. ${ }^{2}$ This survey attested that a younger age $(<4$ years old $)$ at initial presentation was significantly as-
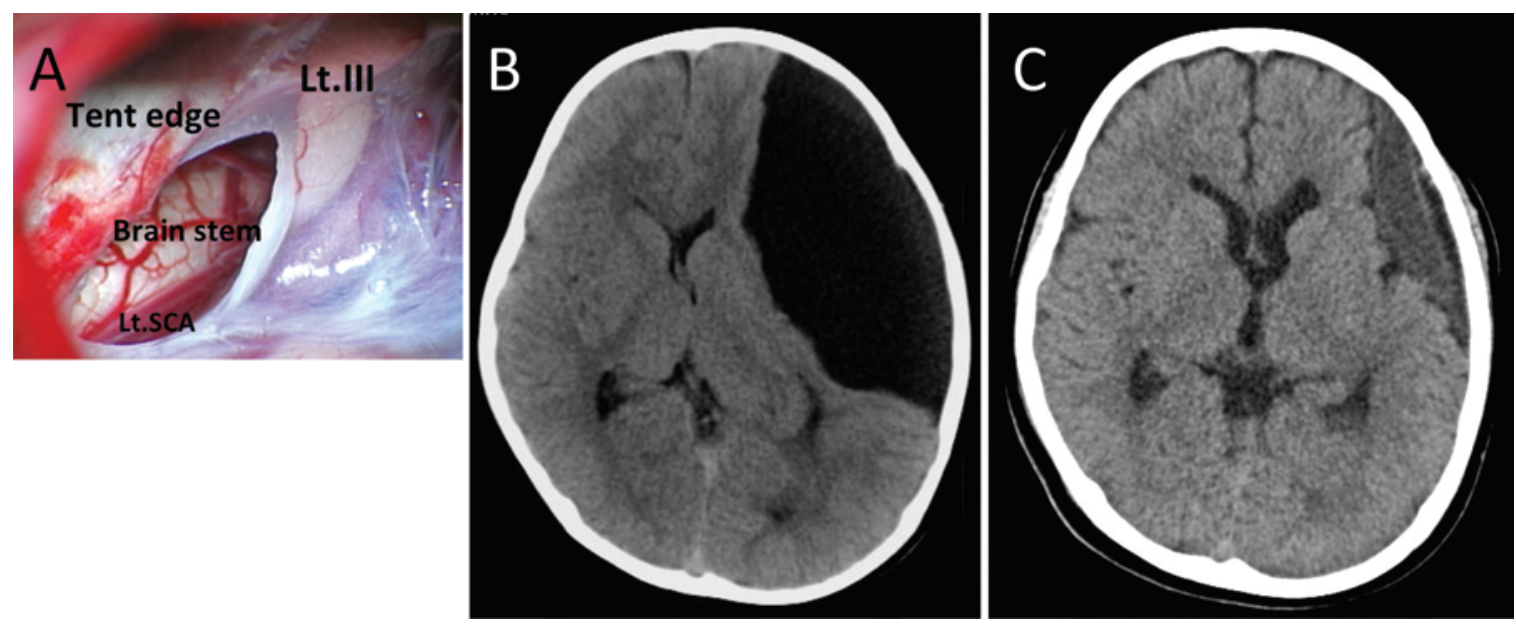

FIG. 5. Case 18. A: A microsurgical intraoperative view of an asymptomatic 8-year-old boy who underwent a successful cyst fenestration. Lt. III = left cranial nerve III; Lt. SCA = left superior cerebellar artery. B: Preoperative axial CT image illustrating a large left middle cranial fossa arachnoid cyst (Galassi Type III). C: Axial CT image after cyst fenestration. Note that the arachnoid cyst was strikingly reduced in size after fenestration. Figure is available in color online only. 
sociated with cyst enlargement, and a larger initial cyst size was related to the presence or development of symptoms and the need for surgery. In contrast, Lee et al. also identified a younger age at diagnosis to be the only significant factor affecting cyst enlargement, but their findings indicated that children with asymptomatic enlargement of arachnoid cysts should not undergo surgery because the risks and complications of surgery were not negligible, adding that close follow-up was an option for such children. ${ }^{3,10}$ Because progressive cyst enlargement and abnormal head enlargement are more likely to occur in younger children, it is possible that this age group can be the only determining factor and at higher risk for shunt placement after fenestration. Because of the small sample size, multivariate analysis could not be performed in this study.

\section{Cyst Reduction After Treatment and Surgical Indication}

The initial treatment in our study was successful, as the middle cranial fossa arachnoid cysts decreased in size or almost disappeared in 22 patients $(84.6 \%)$ after fenestration and/or shunt placement. There were no significant differences in cyst reduction between symptomatic and asymptomatic patients. Regarding the 5 asymptomatic patients with large cysts, the arachnoid cysts had almost disappeared in 3 patients after the fenestration, whereas they had decreased in size in the remaining patients, suggesting that the arachnoid cysts compressed adjacent neural structures even if they appeared to be asymptomatic. Di Rocco et al. implanted ICP sensors in the brain parenchyma adjacent to the cysts in patients and recorded preoperative ICP for 48 hours. They reported that ICP is almost constantly elevated in children with Galassi Type III cysts. ${ }^{5}$ Their results indicate that the adjacent brain parenchyma is locally compressed by arachnoid cysts.

There are no guidelines for the treatment of arachnoid cysts, and most authors agree with treating symptomatic patients with hydrocephalus, elevated ICP, and obvious focalizing symptoms. However, clinical judgment can be difficult when nonspecific symptoms such as headaches, psychosis, or seizures are present. In some cases, the clinician may suspect that the cyst is causing nonspecific difficulties such as developmental delay or other cognitive problems. Wester et al. proposed the possibility that the impairment of higher cognitive functions is reversible after surgical cyst decompression. ${ }^{8,17}$ As described previously, it is unclear whether local compression of the underlying cerebral parenchyma by arachnoid cysts can have adverse effects on brain functions and development. Certainly, it is difficult to investigate the functional and developmental impairments caused by chronic local brain compression due to arachnoid cysts, but we can consider the potential of prophylactic treatment for large asymptomatic arachnoid cysts such as Galassi Type III lesions to protect the developing brain in pediatric patients. In addition to the consensus that symptomatic arachnoid cysts should be treated, we believe that, in children, arachnoid cysts documented as causing significant displacement of normal brain structures warrant surgical treatment to protect brain development.

Children with arachnoid cysts are also at risk for intracystic or subdural hemorrhage occurring spontaneously or after a minor head injury.114 A more recent publication by Cress et al. involved a case-control study to determine whether an association exists among the rupture of arachnoid cysts, cyst size, and recent head injury. These authors found that $6 \%$ of arachnoid cysts presented together with a rupture or hemorrhage, and larger cyst size $(>5 \mathrm{~cm})$ and a recent history of head trauma were significantly associated with arachnoid cyst rupture or hemorrhage. ${ }^{4}$ However, it remains controversial whether surgical interventions can prevent arachnoid cyst rupture/hemorrhage.

Finally, the indication of surgical treatment for intracranial arachnoid cysts remains unresolved. Further studies are needed to clarify whether surgical interventions can preserve brain development in pediatric patients with asymptomatic arachnoid cysts.

\section{Conclusions}

This study demonstrated that children with middle cranial fossa arachnoid cysts were at increased risk of shunt placement following cyst fenestration, particularly if they were young and displayed head enlargement and cyst expansion before fenestration. It is important to consider whether cyst fenestration will be effective in patients with these risk factors.

\section{References}

1. Aicardi J, Bauman F: Supratentorial extracerebral cysts in infants and children. J Neurol Neurosurg Psychiatry 38: 57-68, 1975

2. Al-Holou WN, Yew AY, Boomsaad ZE, Garton HJ, Muraszko KM, Maher CO: Prevalence and natural history of arachnoid cysts in children. J Neurosurg Pediatr 5:578-585, 2010

3. Choi JW, Lee JY, Phi JH, Kim SK, Wang KC: Stricter indications are recommended for fenestration surgery in intracranial arachnoid cysts of children. Childs Nerv Syst 31:77-86, 2015

4. Cress M, Kestle JR, Holubkov R, Riva-Cambrin J: Risk factors for pediatric arachnoid cyst rupture/hemorrhage: a casecontrol study. Neurosurgery 72:716-722, 2013

5. Di Rocco C, Tamburrini G, Caldarelli M, Velardi F, Santini P: Prolonged ICP monitoring in Sylvian arachnoid cysts. Surg Neurol 60:211-218, 2003

6. El-Ghandour NM: Endoscopic treatment of middle cranial fossa arachnoid cysts in children. J Neurosurg Pediatr 9:231-238, 2012

7. Gangemi M, Seneca V, Colella G, Cioffi V, Imperato A, Maiuri F: Endoscopy versus microsurgical cyst excision and shunting for treating intracranial arachnoid cysts. J Neurosurg Pediatr 8:158-164, 2011

8. Gjerde PB, Schmid M, Hammar A, Wester K: Intracranial arachnoid cysts: impairment of higher cognitive functions and postoperative improvement. J Neurodev Disord 5:21, 2013

9. Kaliaperumal C, O'Connor B, Marks C: Development of intracranial hypertension after surgical management of intracranial arachnoid cyst: report of three cases and review of the literature. World Neurosurg 80:222.e1-222.e4, 2013

10. Lee JY, Kim JW, Phi JH, Kim SK, Cho BK, Wang KC: Enlarging arachnoid cyst: a false alarm for infants. Childs Nerv Syst 28:1203-1211, 2012

11. Levy ML, Meltzer HS, Hughes S, Aryan HE, Yoo K, Amar AP: Hydrocephalus in children with middle fossa arachnoid cysts. J Neurosurg 101 (1 Suppl):25-31, 2004 
12. Levy ML, Wang M, Aryan HE, Yoo K, Meltzer H: Microsurgical keyhole approach for middle fossa arachnoid cyst fenestration. Neurosurgery 53:1138-1145, 2003

13. Li C, Yin L, Jiang T, Ma Z, Jia G: Shunt dependency syndrome after cystoperitoneal shunting of arachnoid cysts. Childs Nerv Syst 30:471-476, 2014

14. Peyser E, Weissberg D: Post-traumatic arachnoidal cyst. Report of an unusual case. J Neurosurg 18:551-553, 1961

15. Raffel C, McComb JG: To shunt or to fenestrate: which is the best surgical treatment for arachnoid cysts in pediatric patients? Neurosurgery 23:338-342, 1988

16. Silav G, Sari R, Bolukbasi FH, Altas M, Isik N, Elmaci I: Microsurgical fenestration and cystoperitoneal shunt through preauricular subtemporal keyhole craniotomy for the treatment of symptomatic middle fossa arachnoid cysts in children. Childs Nerv Syst 31:87-93, 2015

17. Wester K, Hugdahl K: Arachnoid cysts of the left temporal fossa: impaired preoperative cognition and postoperative improvement. J Neurol Neurosurg Psychiatry 59:293-298, 1995
18. Zhang B, Zhang Y, Ma Z: Long-term results of cystoperitoneal shunt placement for the treatment of arachnoid cysts in children. J Neurosurg Pediatr 10:302-305, 2012

\section{Author Contributions}

Conception and design: Kimiwada. Acquisition of data: Kimiwada. Analysis and interpretation of data: Kimiwada. Drafting the article: Kimiwada. Critically revising the article: Kimiwada, Hayashi. Reviewed submitted version of manuscript: Kimiwada, Hayashi. Approved the final version of the manuscript on behalf of all authors: Kimiwada. Statistical analysis: Kimiwada. Administrative/technical/material support: Hayashi. Study supervision: Hayashi, Narisawa, Shirane, Tominaga.

\section{Correspondence}

Tomomi Kimiwada, Department of Neurosurgery, Miyagi Children's Hospital, 4-3-17 Ochiai, Aobaku, Sendai 989-3126, Japan. email: tomomi@nsg.med.tohoku.ac.jp. 